\title{
An important minority of prediabetic first-degree relatives of type 1 diabetic patients derives from seroconversion to persistent autoantibody positivity after 10 years of age
}

\author{
I. Vermeulen • I. Weets $\cdot$ O. Costa $\cdot$ M. Asanghanwa $\cdot$ \\ K. Verhaeghen $\cdot$ K. Decochez $\cdot$ J. Ruige $\cdot$ K. Casteels $\cdot$ \\ J. Wenzlau • J. C. Hutton • D. G. Pipeleers • \\ F. K. Gorus • the Belgian Diabetes Registry
}

Received: 8 July 2011 / Accepted: 14 October 2011 / Published online: 18 November 2011

(C) Springer-Verlag 2011

\begin{abstract}
Aims/hypothesis The appearance of autoantibodies (Abs) before diabetes onset has mainly been studied in young children. However, most patients develop type 1 diabetes after the age of 15 years. In first-degree relatives aged under 40 years, we investigated the frequency of seroconversion to (persistent) Ab positivity, progression to diabetes and baseline characteristics of seroconverters according to age.
\end{abstract}

Electronic supplementary material The online version of this article (doi:10.1007/s00125-011-2376-1) contains peer-reviewed but unedited supplementary material, including a list of the current members of the Belgian Diabetes Registry, which is available to authorised users.

I. Vermeulen $\cdot$ I. Weets $\cdot$ M. Asanghanwa $\cdot$ K. Decochez $\cdot$

D. G. Pipeleers $\cdot$ F. K. Gorus $(\triangle)$

Diabetes Research Center, Brussels Free University, VUB,

Laarbeeklaan 103,

1090 Brussels, Belgium

e-mail: frans.gorus@uzbrussel.be

I. Weets $\cdot$ O. Costa $\cdot$ K. Verhaeghen $\cdot$ F. K. Gorus

Department of Clinical Chemistry and Radio-immunology,

University Hospital Brussels Free University, UZ Brussel,

Brussels, Belgium

\section{J. Ruige}

Department of Endocrinology, University of Ghent,

Ghent, Belgium

K. Casteels

Department of Endocrinology, LEGENDO,

Catholic University of Leuven,

Leuven, Belgium

J. Wenzlau $\cdot$ J. C. Hutton

Barbara Davis Center for Childhood Diabetes,

University of Colorado at Denver,

Aurora, CO, USA
Methods Abs against insulin (IAA), glutamate decarboxylase (GADA), insulinoma-associated protein 2 (IA-2A) and zinc transporter 8 (ZnT8A) were measured during follow-up of 7,170 first-degree relatives.

Results We identified 379 (5.3\%) relatives with positivity for IAA, GADA, IA-2A and/or ZnT8A $\left(\mathrm{Ab}^{+}\right)$at first sampling and $224(3.1 \%)$ at a later time point. Most seroconversions occurred after the age of 10 years $(63 \%)$. During follow-up, Abs persisted more often in relatives initially $\mathrm{Ab}^{+}(76 \%)$ than in seroconverters $(53 \%$; $p<0.001)$. In both groups diabetes developed at a similar pace and almost exclusively with $\mathrm{Ab}$ persistence (136 of 139 prediabetic individuals). For both groups, progression was more rapid if Abs appeared before the age of 10 years. Baseline characteristics at seroconversion did not vary significantly according to age.

Conclusions/interpretation Seroconversion to (persistent) $\mathrm{Ab}^{+}$occurs regardless of age. Although the progression rate to diabetes is higher under age 10 years, later seroconverters (up to age 40 years) have similar characteristics when compared with age-matched initially $\mathrm{Ab}^{+}$ relatives and generate an important minority of prediabetic relatives, warranting their identification and, eventually, enrolment in prevention trials.

Keywords Autoantibodies - First-degree relatives - GAD . IA-2 - Insulin · Prediabetes · Proinsulin · Seroconversion . Type 1 diabetes $\cdot$ Zinc transporter 8

\author{
Abbreviations \\ $\mathrm{Ab} \quad$ Autoantibody \\ $\mathrm{Ab}^{+} \quad$ Positivity for IAA, GADA, IA-2A and/or ZnT8A \\ $\mathrm{Ab}^{-} \quad$ Negativity for IAA, GADA, IA-2A and ZnT8A \\ BDR Belgian Diabetes Registry
}


CRCW Hybrid ZnT8 construct generated by fusion of $\mathrm{CR}$ and $\mathrm{CW}$ (zinc transporter-8 carboxy-terminal constructs carrying respectively $325 \mathrm{Arg}$ and $325 \operatorname{Trp})$

GADA Glutamate decarboxylase autoantibodies

IAA Insulin autoantibodies

IA-2 Insulinoma-associated protein 2

IA-2A IA-2 autoantibodies

ICA Islet cell cytoplasmic antibodies

IQR Interquartile range

ZnT8 Zinc transporter 8

ZnT8A ZnT8 autoantibodies

\section{Introduction}

Type 1 diabetes is a heterogeneous disease in terms of underlying pathological process, clinical presentation and biological markers [1-3]. Several clinical studies in recentonset patients have provided proof of principle that selective immuno-interventions may preserve at least temporarily residual beta cell function in subgroups of patients [4, 5]. They have also indicated that future immunomodulation trials should be targeting the preclinical stage, at which beta cell function is better preserved $[5,6]$. Such secondary prevention studies are, however, complicated by our incomplete knowledge of the natural history of pre-type 1 diabetes, in particular for the majority of patients who develop the disease after the age of 15 years [7,8]. Indeed, while several studies have followed newborn infants with or without family history of type 1 diabetes to study the appearance of different autoantibodies (Abs) and their relationship to the development of hyperglycaemia [9-13], data on the frequency of seroconversion to positivity for insulin Abs (IAA), glutamate decarboxylase Abs (GADA), insulinoma-associated protein 2 Abs (IA-2A) and/or zinc transporter $8 \mathrm{Abs}(\mathrm{ZnT} 8 \mathrm{~A})\left(\mathrm{Ab}^{+}\right)$and the subsequent risk of diabetes in older children are scarce and data in young adults virtually absent $[14,15]$. However, more knowledge on the latter topic is warranted as the launch of immuno-intervention trials in pre-type 1 diabetes will require the screening of large groups of participants in order to enrol sufficient numbers of high-risk individuals. In this context, it is important to know whether it is relevant to continue screening adolescents and young adults who have previously tested negative for Abs.

In the present study, we followed and repeatedly sampled a large representative group of first-degree relatives, initially with negativity for IAA, GADA, IA-2A and $\mathrm{ZnT} 8 \mathrm{~A}$ ( $\mathrm{Ab}^{-}$; aged 0-39 years), of patients with type 1 diabetes recruited by the Belgian Diabetes Registry (BDR). The aims were to detect individuals who seroconverted to $\mathrm{Ab}^{+}$, to estimate the frequency of this phenomenon over a wider age range than previously studied and to investigate the baseline characteristics of seroconverters and their progression to diabetes according to age at seroconversion in comparison with initially $\mathrm{Ab}^{+}$relatives.

\section{Methods}

Participants Between August 1989 and January 2010, the BDR consecutively recruited 9,040 siblings, offspring or parents (under age 40 years at entry) of type 1 diabetic probands according to previously defined criteria. The probands are considered representative of the Belgian population of type 1 diabetic patients [7]. After obtaining written informed consent from each relative or their parents, a short questionnaire with demographic, familial and personal information was completed at each visit and blood samples were taken at entry and, as a rule, yearly thereafter. Relatives who were $\mathrm{Ab}^{-}$on four consecutive yearly visits were re-invited 4 years later. Only relatives with two or more contacts during follow-up $(7,170$ of 9,040 ), the last being at diagnosis in the case of prediabetes, were included in this study. This allowed unambiguous ascertainment of the clinical status of relatives at this last time point.

The study was conducted in accordance with the guidelines in the Declaration of Helsinki as revised in 2008 (www.wma. net/en/30publications/10policies/b3/index.html, accessed 8 September 2011) and approved by the ethics committees of the BDR and the participating university hospitals. Random blood samples were collected for sera, plasma and buffy coats, and aliquots were stored at $-80^{\circ} \mathrm{C}$ until analysed for diabetes-associated autoantibodies, hormonal markers and $H L A-D Q$ genotype, respectively, as previously described [16]. Relatives were longitudinally screened for the presence of IAA, GADA and IA-2A. Individuals who had at least one $\mathrm{Ab}^{-}$sample before the development of IAA, GADA and/or IA-2A - seroconverters - were all sampled at least once after seroconversion to $\mathrm{Ab}^{+}$. $\mathrm{ZnT} 8 \mathrm{~A}$ were also determined in all samples from these relatives. Relatives were not pre-screened for islet cell cytoplasmic antibodies (ICA), nor were ICA results analysed in the present study. During follow-up of initially $\mathrm{Ab}^{-}$first-degree relatives, the moment of seroconversion was approximated from the sampling time of the serum for which positivity for at least one Ab type was first detected. Both in seroconverters and in initially $\mathrm{Ab}^{+}$ relatives, $\mathrm{Ab}^{+}$was defined as transient if the next sample was negative for all Abs; it was defined as persistent if the next sample was positive for at least one Ab type. The median (interquartile range [IQR]) of the time between the last $\mathrm{Ab}^{-}$and the first $\mathrm{Ab}^{+}$sample was 13 months (1224 months). During follow-up, development of diabetes was ascertained through repeated contacts with Belgian endocri- 
nologists and paediatricians, self-reporting through yearly questionnaires and a link with the BDR patient database, where newly diagnosed patients under 40 years of age are registered. Follow-up ended at the time of the last blood sampling or, in the case of prediabetes, at clinical onset.

Analytical methods IAA, GADA, IA-2A and ZnT8A were determined by liquid-phase radiobinding assays [16], C-peptide by time-resolved fluorescence immunoassay [17], and HLA-DQ polymorphisms by allele-specific oligonucleotide genotyping [18], as described previously. The tracers for the $A b$ assays were purified by ultrafiltration (Amicon Ultra-4 filter units; Millipore, MA, USA) in the case of in vitro transcription/translation or by gel exclusion chromatography for A14- ${ }^{125}$ I-labelled insulin. Ab levels were expressed as percentages of bound added tracer $(10,000 \mathrm{cpm} /$ tube $)$. cDNAs for the preparation of radioligands by in vitro transcription-translation were kind gifts of A. Lernmark (when at University of Washington, Seattle, WA, USA) for full-length $65 \mathrm{kDa}$ glutamate decarboxylase, M. Christie (King's College School of Medicine and Dentistry, London, UK) for the intracellular portion of insulinoma-associated protein 2 (IA-2) and J. C. Hutton (Barbara Davis Center for Childhood Diabetes, Aurora, CO, USA) for the dimeric hybrid ZnT8 construct generated by fusion of $\mathrm{CR}$ and $\mathrm{CW}$ (zinc transporter-8 carboxy-terminal constructs carrying, respectively, Arg325 and Trp325) (CRCW). In the 2009 Diabetes Autoantibody Standardisation Program (DASP) Workshop diagnostic sensitivity and specificity were, respectively, $74 \%$ and $97 \%$ for GADA, $40 \%$ and $98 \%$ for IAA, $66 \%$ and $99 \%$ for IA-2A and $68 \%$ and $100 \%$ for ZnT8A (CRCW). Cut-off values for $\mathrm{Ab}^{+}$were determined as the 99th percentile of Ab levels in 761 nondiabetic controls, and amounted to $\geq 0.6 \%$ tracer binding for IAA, $\geq 2.6 \%$ for GADA, $\geq 0.44 \%$ for IA- $2 \mathrm{~A}$ and $\geq 1.20 \%$ for ZnT8A. Between-day coefficients of variation determined for serum pools within the normal range and within the moderately elevated range were, respectively, 35\% $(0.3 \%$ tracer binding) and $12 \%$ (6.9\% tracer binding) for IAA, $12 \%$ (2.1\% tracer binding) and $10 \%$ (7.1\% tracer binding) for GADA, $18 \%$ ( $0.3 \%$ tracer binding) and $9 \%(2.3 \%$ tracer binding) for IA-2A, and $21 \%(0.7 \%$ tracer binding $)$ and $6 \%$ (3.9\% tracer binding) for ZnT8A.

Statistical analysis The statistical significance of differences between groups was assessed by the $\chi^{2}$ test, with Yates' correction or Fisher's exact test for categorical variables and by Mann-Whitney $U$ test for continuous variables. To estimate diabetes-free survival, Kaplan-Meier analysis was used; the survival curves were compared using the logrank test. In time-to-event analysis, follow-up started at the time of the first $\mathrm{Ab}^{+}$sample and ended at the last contact with the relative or at clinical onset, whichever came first. All statistical tests were performed two-tailed by SPSS for Windows 16.0 (SPSS, Chicago, IL, USA) or by GraphPad Prism version 5.00 for Windows (San Diego, CA, USA) and considered significant at $p<0.05$ or $p<0.05 / k$ in case of multiple comparisons (Bonferroni correction).

\section{Results}

Seroconversion to $\mathrm{Ab}^{+}$and progression to diabetes according to age Among the 7,170 first-degree relatives aged 0-39 years at inclusion and followed for a median (IQR) period of 60 (36-109) months, $603(8.4 \%)$ tested positive at least once for one or more types of $\mathrm{Ab}$ (i.e. $\mathrm{IAA}^{+}, \mathrm{GADA}^{+}$,

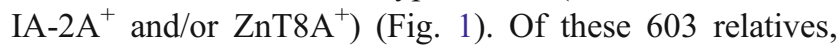
379 (5.3\% of all relatives) were positive at first sampling. Their median (IQR) age was 13 (7-24) years and the male/ female ratio $185 / 194(0.95 ; p>0.05$ vs 0.95 in all relatives). The remaining $224(3.1 \%)$ relatives seroconverted at a median (IQR) age of 13 (7-22) years (male/female ratio: $123 / 101$ or $1.22 ; p>0.05 \mathrm{vs}$ in all relatives) after a median (IQR) follow-up of 27 (15-52) months. The median (IQR) time between their last $\mathrm{Ab}^{-}$sample before seroconversion and the first $\mathrm{Ab}^{+}$sample was 13 (12-24) months. Overall, $139 / 603(23 \%) \mathrm{Ab}^{+}$relatives developed diabetes to date at a median (IQR) age of 14 (10-23) years and after a median (IQR) follow-up of 44 (20-82) months, including 109 (29\%) of the 379 relatives who were $\mathrm{Ab}^{+}$at baseline and 30 $(13 \%)$ of the 224 seroconverters to $\mathrm{Ab}^{+}$. Three $(0.05 \%)$ of the 6,567 persistently $\mathrm{Ab}^{-}$relatives also developed diabetes (Fig. 1).

Persistent vs transient $\mathrm{Ab}^{+}$according to age The prevalence of initially $\mathrm{Ab}^{+}$relatives ranged between $4.8 \%$ and $5.9 \%$ according to age $(p>0.05)$ and most were persistently $\mathrm{Ab}^{+}$(289/379 or $\left.76 \%\right)$, regardless of age (Table 1). In initially $\mathrm{Ab}^{-}$relatives most seroconversions (141 of 224 or

First-degree relatives enrolled between 1989 and 2010 with $\geq 2$ visits for Ab screening

$\mathrm{Ab}^{+}$in $\geq 1$ sample

$\mathrm{Ab}^{+}$in 1 st sample

Diabetes

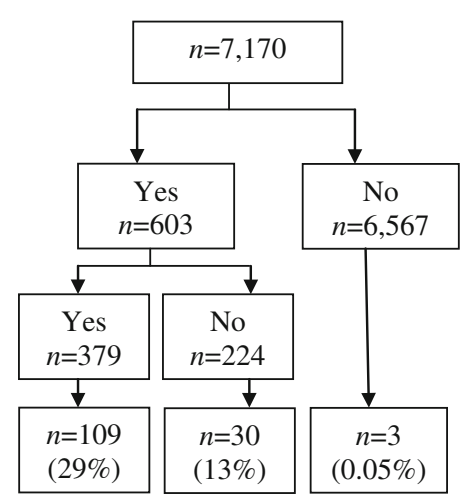

Fig. 1 Disposition diagram showing all participants in the present study, their $\mathrm{Ab}$ status and their progression to diabetes 
Table 1 Frequency of seroconversion to $\mathrm{Ab}^{+}$and development of diabetes according to age at seroconversion
Threshold for significance: overall $p<0.05 / 18$ or $p<0.0028$; for differences among age groups: $p<0.05 / 3$ or $p<0.017$ (Bonferroni correction). ${ }^{\mathrm{a}}$ Overall $p=0.020 ;{ }^{\mathrm{b}}$ overall $p=0.042$; ${ }^{\mathrm{c}}$ overall $p<0.001 ;{ }^{\mathrm{d}} p=0.015$; ${ }^{\mathrm{e}} p<0.001 ;{ }^{\mathrm{f}} p<0.010$ vs age group $0-9$ years; ${ }^{\mathrm{g}}$ overall $p=0.027 ;{ }^{h}$ Follow-up time also limited by age limit

\begin{tabular}{|c|c|c|c|}
\hline \multirow[t]{2}{*}{ Relatives } & \multicolumn{3}{|l|}{ Age group } \\
\hline & $0-9$ years & 10-19 years & 20-39 years \\
\hline Total number $(n)$ & 2,581 & 2,055 & 2,534 \\
\hline Initially $\mathrm{Ab}^{-}(n)$ & 2,444 & 1,934 & 2,413 \\
\hline \multicolumn{4}{|l|}{$\mathrm{Ab}^{+}$relatives } \\
\hline $\mathrm{Ab}^{+}$at baseline, $n$ ( $\%$ of total) & $137(5.3)$ & $121(5.9)$ & $121(4.8)$ \\
\hline Persistently $\mathrm{Ab}^{+}, n(\%$ of total $)$ & $105(4.1)$ & $94(4.6)$ & $90(3.6)$ \\
\hline Transiently $\mathrm{Ab}^{+}, n(\%$ of total $)$ & $32(1.2)$ & $27(1.3)$ & $31(1.2)$ \\
\hline Seroconverters, $n\left(\% \text { of initially } \mathrm{Ab}^{-}\right)^{\mathrm{a}}$ & $83(3.4)$ & $79(4.1)$ & $62(2.6)$ \\
\hline Persistently $\mathrm{Ab}^{+}, n\left(\% \text { of initially } \mathrm{Ab}^{-}\right)^{\mathrm{b}}$ & $50(2.0)$ & $39(2.0)$ & $29(1.2)$ \\
\hline Transiently $\mathrm{Ab}^{+}, n\left(\%\right.$ of initially $\left.\mathrm{Ab}^{-}\right)$ & $33(1.4)$ & $40(2.1)$ & $33(1.4)$ \\
\hline \multicolumn{4}{|l|}{ Diabetes } \\
\hline $\mathrm{Ab}^{+}$at baseline, $n$ (\% of group $)^{\mathrm{c}}$ & $56(41)$ & $32(26)^{\mathrm{d}}$ & $21(17)^{\mathrm{e}}$ \\
\hline Persistently $\mathrm{Ab}^{+}, n$ (\% of group $)^{\mathrm{c}}$ & $56(53)$ & $32(34)^{\mathrm{f}}$ & $20(22)^{d}$ \\
\hline Transiently $\mathrm{Ab}^{+}, n$ (\% of group) & $0(0)$ & $0(0)$ & $1(3.2)$ \\
\hline Seroconverters, $n$ ( $\%$ of group $)^{\mathrm{g}}$ & $18(22)$ & $7(8.9)$ & $5(8.1)$ \\
\hline Persistently $\mathrm{Ab}^{+}, n(\% \text { of group })^{\mathrm{g}}$ & $18(36)$ & $6(15)$ & $4(14)$ \\
\hline Transiently $\mathrm{Ab}^{+}, n$ (\% of group) & $0(0)$ & $1(2.5)$ & $1(3.0)$ \\
\hline \multicolumn{4}{|l|}{ Median $(\mathrm{IQR})^{\mathrm{h}}$ follow-up from $\mathrm{Ab}^{+}$} \\
\hline $\mathrm{Ab}^{+}$at baseline (months) & $74(43-124)$ & $77(36-124)$ & $62(38-112)$ \\
\hline Persistently $\mathrm{Ab}^{+}$(months) & $64(37-110)$ & $62(36-115)$ & $61(37-122)$ \\
\hline Transiently $\mathrm{Ab}^{+}$(months) & $107(61-140)$ & $113(71-139)$ & $64(48-100)^{\mathrm{h}}$ \\
\hline Seroconverters, $n$ (months) $^{\mathrm{b}}$ & $59(25-92)$ & $48(20-96)$ & $59(24-89)$ \\
\hline Persistently $\mathrm{Ab}^{+}$(months) & $53(21-84)$ & $47(16-96)$ & $60(14-115)$ \\
\hline Transiently $\mathrm{Ab}^{+}$(months) & $72(34-100)$ & $49(23-96)$ & $59(25-88)$ \\
\hline
\end{tabular}

$63 \%$ ) occurred after the age of 10 years. The frequency ranged between $2.6 \%$ and $4.1 \%$ but did not vary significantly according to age (Table 1); this was also the case when 5-year age groups were considered (Electronic supplementary material [ESM] Fig. 1). Overall, 53\% of seroconverters developed persistent $\mathrm{Ab}^{+}(p<0.001$ vs $76 \%$ in initially $\mathrm{Ab}^{+}$relatives). This fraction tended to decrease from $60 \%$ under age 10 years to $48 \%$ after age 20 years, without reaching significance (Table 1). In both initially $\mathrm{Ab}^{+}$relatives $(108 / 109$ or $99 \%)$ and seroconverters $(28 / 30$ or $93 \%$ ) progression to diabetes occurred almost exclusively in persistently $\mathrm{Ab}^{+}$relatives (Table 1 and ESM Fig. 1). Therefore, follow-up time from $\mathrm{Ab}^{+}$was shorter in this group compared with transiently $\mathrm{Ab}^{+}$relatives (Table 1). Progression to diabetes tended also to be more frequent in those seroconverting before the age of 10 years (Table 1 and ESM Fig. 1). This was not due to differences in follow-up time according to age (Table 1). Among the 109 initially $\mathrm{Ab}^{+}$ relatives who progressed to diabetes, at least 56 (51\%) had developed Abs before age 10 years compared with 18 of 30 $(60 \%)$ prediabetic seroconverters (Table 1$)$. Two relatives who developed initially transient $\mathrm{Ab}^{+}$at ages 12 and 24 years, respectively, progressed to diabetes 7 and 8 years later, respectively, but not before having become $\mathrm{Ab}^{+}$again at an undefined later time point (Table 1). In 21 of the 30 prediabetic seroconverters $(70 \%)$, diabetes was diagnosed after age $10 ; 12$ of these $21(57 \%)$ had seroconverted after age 10 years and 5 of them (24\%) after age 20 years (data not shown).

After seroconversion to persistent $\mathrm{Ab}^{+}$, progression to diabetes occurred at a pace that was not significantly different from that in initially $\mathrm{Ab}^{+}$relatives (Fig. 2a). In both groups the progression rate decreased with age at first $\mathrm{Ab}^{+}$(Fig. 2b, c).

Baseline characteristics of seroconverters to persistent $\mathrm{Ab}^{+}$ according to age Overall, there was a tendency towards a male excess in the seroconverters (male/female ratio: 69/49 or $1.41 ; p=0.044$ vs 0.95 in all relatives). As shown in Table 2, the male/female ratio tended to decrease with age at seroconversion but significance was lost after correction for multiple comparisons; compared with age-matched $\mathrm{Ab}^{-}$ relatives the ratio was only higher for seroconversion under age 10 years $(2.33$ vs $1.14 ; p=0.027)$. Young seroconverters more often tended to be $\mathrm{IAA}^{+}, \mathrm{IA}_{-}-2 \mathrm{~A}^{+}$and/or $\mathrm{ZnT}_{8} \mathrm{~A}^{+}$, but in the case of $\mathrm{Ab}^{+}$, circulating levels did overall not differ according to age (Table 2). During followup of persistently $\mathrm{Ab}^{+}$relatives there was no fixed sequence 

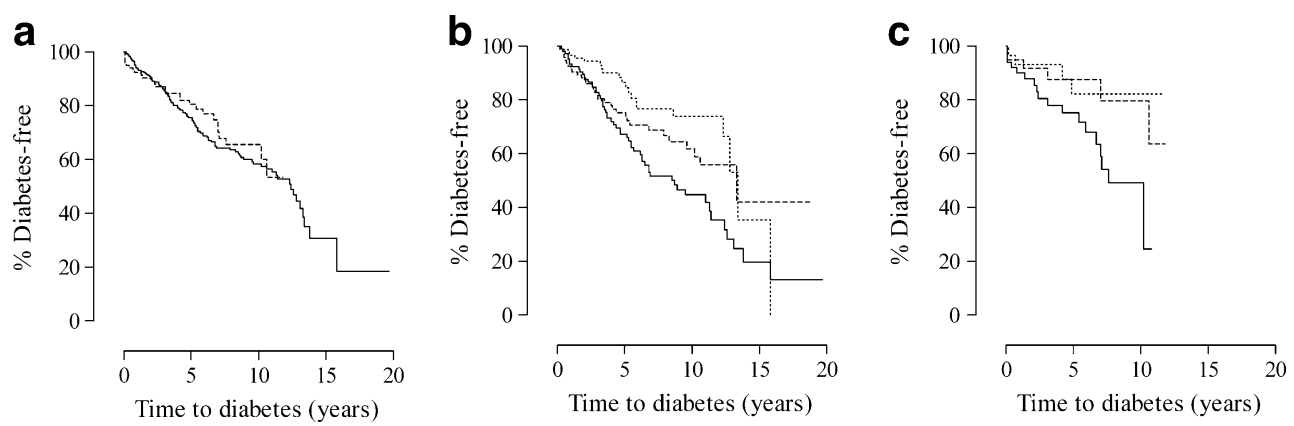

Fig. 2 Diabetes-free survival of persistently $\mathrm{Ab}^{+}$relatives as a function of time after first $\mathrm{Ab}^{+}$sample. a $\mathrm{Ab}^{+}$relatives at baseline (solid line, $n=289$ at time 0 ) vs seroconverters to $\mathrm{Ab}^{+}$(dashed line, $n=118$ at time 0 ) ( $p=0.505$ by logrank). b $\mathrm{Ab}^{+}$relatives at baseline according to age at inclusion (solid line, 0-9 years; dashed line,

for the appearance of the various types of $\mathrm{Ab}$ (data not shown), but IA-2A and ZnT8A tended to develop more often after the first biological evidence of the autoimmune process (up to more than 7 years later; ESM Fig. 2) in all age categories (data not shown).

\section{Discussion}

During follow-up of over 7,000 first-degree relatives of patients with type 1 diabetes we identified $379 \mathrm{Ab}^{+}$
10-19 years; dotted line, 20-39 years; $p=0.003$ by logrank). c Seroconverters to $\mathrm{Ab}^{+}$according to age at seroconversion (solid line, $0-9$ years; dashed line, $10-19$ years; dotted line, 20-39 years; $p=0.042$ by logrank)

relatives at first sampling and 224 individuals who seroconverted to islet $\mathrm{Ab}^{+}$. Our main finding was that seroconversion can occur at any age between 0 and 40 years at a rate that does not significantly differ according to age, with most events occurring after age 10. During follow-up, Abs persisted more often in initially $\mathrm{Ab}^{+}$relatives than in seroconverters. Both groups developed diabetes at a similar pace and almost exclusively in the presence of $\mathrm{Ab}$ persistence. For both groups, progression was more rapid if Abs were first detected under the age of 10 years. The baseline characteristics of relatives at seroconversion did not differ greatly
Table 2 Demographic and biological baseline characteristics of first-degree relatives who seroconverted to persistent $\mathrm{Ab}^{+}$according to age at seroconversion
Threshold for significance: overall $p<0.05 / 14$ or $p<0.0037$, for differences among age groups: $p<0.05 / 3$ or $p<0.017$ (Bonferroni correction).

${ }^{\mathrm{a} O v e r a l l ~} p=0.043 ;{ }^{\mathrm{b}}$ overall $p=0.002$; ${ }^{c}$ overall $p<0.030$

${ }^{\mathrm{d}}$ Only in relatives positive for that particular $\mathrm{Ab}$

NA, not applicable; $\mathrm{P}_{50}$, median

\begin{tabular}{|c|c|c|c|}
\hline \multirow[t]{2}{*}{ Characteristic at seroconversion } & \multicolumn{3}{|c|}{ Persistently $\mathrm{Ab}^{+}$seroconverters $(n=118)$} \\
\hline & $0-9$ years & 10-19 years & 20-39 years \\
\hline$n(\%)$ & $50(42)$ & $39(33)$ & $29(25)$ \\
\hline Age (years), $\mathrm{P}_{50}$ (IQR) & $6(4-8)$ & $14(12-16)$ & $29(22-34)$ \\
\hline Sex, $n$ males $/ n$ females (ratio) ${ }^{\mathrm{a}}$ & $35 / 15(2.33)$ & 22/17 (1.29) & $12 / 17(0.70)$ \\
\hline \multicolumn{4}{|l|}{ Relationship to type 1 diabetes, $n(\%)^{\mathrm{b}}$} \\
\hline Parent & $0(0)$ & $0(0)$ & $19(31)$ \\
\hline Offspring type 1 diabetes mother & $12(24)$ & $12(31)$ & $6(21)$ \\
\hline Offspring type 1 diabetes father & $16(32)$ & $12(31)$ & $5(17)$ \\
\hline Sibling & $22(44)$ & $15(38)$ & $12(41)$ \\
\hline C-peptide (pmol/l), $\mathrm{P}_{50}$ (IQR) & $645(374-943)$ & $731(539-1,155)$ & $867(583-1,162)$ \\
\hline$H L A-D Q 2 / D Q 8, n(\%)$ & $12(24)$ & $8(20)$ & $6(21)$ \\
\hline \multicolumn{4}{|l|}{ Ab type, $n(\%)$} \\
\hline $\mathrm{IAA}^{+}$ & $26(52)$ & $15(38)$ & $10(34)$ \\
\hline $\mathrm{GADA}^{+}$ & $36(72)$ & $29(74)$ & $23(79)$ \\
\hline $\mathrm{IA}-2 \mathrm{~A}^{+\mathrm{c}}$ & $11(22)$ & $6(15)$ & $0(0)$ \\
\hline $\mathrm{ZnT} \mathrm{A}^{+}$ & $7(14)$ & $6(15)$ & $0(0)$ \\
\hline Multiple $\mathrm{Ab}^{+}, n(\%)^{\mathrm{c}}$ & $21(42)$ & $10(26)$ & $4(14)$ \\
\hline \multicolumn{4}{|l|}{ Ab levels ${ }^{\mathrm{d}}$ (\% tracer-bound), $\mathrm{P}_{50}(\mathrm{IQR})$} \\
\hline $\mathrm{IAA}^{+}$ & $1.3(0.8-2.3)$ & $1.1(0.8-2.0)$ & $0.9(0.8-1.2)$ \\
\hline $\mathrm{GADA}^{+}$ & $9.9(4.8-36.1)$ & $15.1(3.6-137)$ & $7.6(3.6-79.7)$ \\
\hline $\mathrm{IA}-2 \mathrm{~A}^{+}$ & $6.9(1.7-83.4)$ & $50(1.1-82.1)$ & NA \\
\hline $\mathrm{ZnT}_{8} \mathrm{~A}^{+}$ & $5.2(2.5-14.2)$ & $2.4(1.7-12.4)$ & NA \\
\hline
\end{tabular}


according to age. In seroconverters, IA-2A and ZnT8A tended to appear later during the subclinical disease process, compatible with their association with - and prediction of rapid progression to clinical onset [16, 19-21].

The strengths of this study are: (1) its longitudinal nature; (2) the registry-based recruitment of first-degree relatives over a wide age range, when little or no data exist on this topic for adults $[14,15]$; (3) the possibility of comparing the clinical outcome of seroconverters with those of $\mathrm{Ab}^{+}$relatives at first sampling; (4) the confirmation of the glycaemic status at the last follow-up point for each relative; (5) the completeness of hormonal, genetic and immunological data for each participant, including results from a sensitive ZnT8A assay, which has so far only rarely been used in longitudinal studies in risk groups; and (6) the lack of selection bias in the absence of prescreening for ICA.

However, this study also has certain weaknesses. At variance with some other studies in children [8-12] few participants were followed from birth onwards, hence previous transient seroconversions may have been missed. Conceivably, the number of young seroconverters and rapidly progressing prediabetic young children may have been underestimated. However, the fact that seroconversion frequency was largely independent of age and that the rate of progression to diabetes was quite similar to that in agematched initially $\mathrm{Ab}^{+}$relatives supports the significant contribution of seroconversion after age 10 to incident cases of type 1 diabetes in adolescence and young adulthood. The exact fraction of all $139 \mathrm{Ab}^{+}$prediabetic relatives who derived from seroconversion after age 10 cannot be precisely determined from the present study: indeed, while we know that all initially $\mathrm{Ab}^{+}$relatives under age 10 must have seroconverted before that age, we do not know how many of the older initially $\mathrm{Ab}^{+}$relatives derived from late seroconversion. Establishing the precise time of seroconversion and the order of appearance of various $\mathrm{Ab}$ types was limited by the relatively large intervals between successive blood samples. As a rule these intervals approximated 12 months, but in a minority of cases they spanned several years. Therefore, the age at seroconversion may have been overestimated at times. However, our conclusions remained unchanged if the age at positive seroconversion was approximated from the age at the last $\mathrm{Ab}^{-}$sample (not shown).

Our study design may also have been limited by the absolute number of established seroconversions leading to clinical appearance of type 1 diabetes, which may have decreased the power of subgroups analysis. However, because of the size of our registry and the study of firstdegree relatives our numbers of seroconverters compare well with those observed in previous studies in young children or in the general population $[13,14,20]$. One may also criticise the fact that we did not include ICA in our analysis as did some other studies [9, 22]. This was a conscious choice because ICA are not completely independent of GADA, IA-2A and ZnT8A, which are all believed to contribute to ICA reactivity $[19,23,24]$. ZnT8A were only determined in all samples of relatives who tested positive on at least one occasion for other islet Abs, but previous results have shown that their prevalence in relatives lacking the other Abs is virtually zero [16, 25].

To the best of our knowledge, our results are the first to compare seroconversion frequencies for islet $\mathrm{Abs}$ in children, adolescents and adults at familial risk of diabetes. The age independency of the seroconversion frequency warrants regular reassessment of $\mathrm{Ab}$-inferred risk of diabetes in first-degree relatives up to 40 years of age in the context of a further in-depth study of the preclinical phase of type 1 diabetes in adults and of identifying additional potential participants in new secondary prevention trials. Our results are also compatible with previous findings indicating that an early appearance of $\mathrm{Abs}$ and $\mathrm{Ab}$ persistence are preferentially associated with a rapid progression to clinical onset $[9,20,26-30]$. The infrequent progression to diabetes in case of transient $\mathrm{Ab}^{+}$may relate to false-positive results due to the relatively high imprecision of $\mathrm{Ab}$ assays in the decision zone, particularly for IAA (see Methods) or to 'statistical' positivity due to the choice of the 99th percentile as cut-off value. However, in some cases $\mathrm{Ab}$ levels were clearly transiently elevated and may have reappeared later, suggesting that autoimmunity may at times follow a relapsing/remitting pattern and in other instances a more aggressive and progressive course, similar to that reported for other autoimmune diseases such as multiple sclerosis [31]. IAA and GADA were confirmed as relatively early immune markers in prediabetes, but only GADA or multiple $\mathrm{Ab}^{+}$were predictive of $\mathrm{Ab}$ persistence (data not shown). The tendency towards later appearance of IA-2A and ZnT8A is compatible with their reported association with a more rapid progression to diabetes [16, 19, 20, 32-35]. Overall, there was a slight male excess in seroconverters compatible with the male predominance in type 1 diabetes under age 40 [3, 7, 8]; when adjusted for sex ratio in age-matched groups of all included relatives, this male excess was only significant in case of seroconversion under age 10 . The trend towards more multiple $\mathrm{Ab}^{+}$ in those seroconverting under age 10 years is compatible with the more rapid progression to diabetes in this age group [20, 22]. Finally, the seroconversion rates observed for children and adolescents in the present study are similar to recent data from DPT-1 and TrialNet cohorts [36, 37] but the latter studies were limited to children under age 18 , included also second- [36, 37] or third-degree [37] relatives and did not distinguish between seroconversion to transient or persistent $\mathrm{Ab}^{+}$. At variance with these reports, the 
seroconversion rate in our study only tended to decrease after the age of 20 years.

In conclusion, the frequency of seroconversion approximates to $3 \%$ regardless of age in first-degree relatives under age 40 years, with most events occurring after age 10 years. As in initially $\mathrm{Ab}^{+}$relatives, progression rate to diabetes was highest for those seroconverting before age 10 years and occurred almost exclusively in persistently $\mathrm{Ab}^{+}$relatives. However, an important minority of prediabetic relatives derived from seroconversion after age 10 years. As only about $15 \%$ of all new patients have a family history of the disease, further studies should investigate whether our conclusions in relatives also hold for the majority of sporadic cases. Reports that patients with or without familial history of type 1 diabetes have quite similar characteristics $[38,39]$ suggest that this may indeed be the case. Our data on Ab development and persistence should be taken into account when planning further prediction and prevention studies and warrant continued monitoring of $\mathrm{Ab}$ status up to at least 40 years in risk groups such as first-degree relatives.

Acknowledgements This work was presented in part at the 46th EASD meeting, 20-24 September 2010, Stockholm, Sweden. The present work was supported by grants from the Juvenile Diabetes Research Foundation (JDRF Center Grant 4-2005-1327), the European Union (FP-7 project no. 241833), the Belgian Fund for Scientific Research (FWO Vlaanderen projects G.0319.01, G.0514.04, G.0311.07, G.0374.08 and G.0868.11; senior clinical research fellowship for K. Casteels, K. Decochez and I. Weets), the research council of the Brussel Free University (projects OZR1150, 1449 and 1615) and the Willy Gepts Fund (projects 3-2005 and 3/22-2007; University Hospital Brussels - UZ Brussel). J. C. Hutton acknowledges DERC (NIH P30 DK57516), NIH R01 DK052068 and JDRF 4-20071056. The BDR was sponsored by the Belgian National Lottery, the ministries of Public Health of the Flemish and French Communities of Belgium, Weight Watchers, Ortho-Clinical Diagnostics, Novo Nordisk Pharma, Lifescan, Roche Diagnostics, Bayer and Eli Lilly. The expert technical assistance of co-workers at the central unit of the BDR (V. Baeten, G. de Block, T. de Mesmaeker, L. de Pree, H. Dewinter, N. Diependaele, S. Exterbille, P. Goubert, C. Groven, A. Ivens, D. Kesler, F. Lebleu, M. Lichtert, E. Quartier, G. Schoonjans, U. Vandevelde, M. van Molle, S. Vanderstraeten, and A. Walgrave) is gratefully acknowledged. We would also like to thank the different university teams of co-workers for their excellent assistance in collecting samples and organising the fieldwork: in Antwerp (L. van Gaal, C. de Block, J. Michiels, J. van Elven and J. Vertommen); in Brussels (T. de Mesmaeker, S. Exterbille, P. Goubert, C. Groven, M. Lichtert, S. Vanderstraeten and A. Walgrave); in Ghent (J. M. Kaufman, J. Ruige, A. Hutse and A. Rawoens); and in Leuven (C. Mathieu, P. Gillard, M. Carpentier, M. Robijn, K. Rouffé, A. Schoonis and H. Morobé). We sincerely thank all members of the BDR who contributed to the recruitment of relatives for the present study.

Contribution statement IV designed research, acquired, analysed and interpreted data, provided statistical analysis and reviewed/edited the manuscript; IW designed research, recruited first-degree relatives, analysed and interpreted data and reviewed/edited the manuscript; OC, $\mathrm{MA}$ and $\mathrm{KV}$ acquired, analysed and interpreted data and reviewed/ edited the manuscript; $\mathrm{KD}, \mathrm{JR}$, and $\mathrm{KC}$ designed research, recruited first-degree relatives, contributed clinical data and reviewed/edited the manuscript; JW and JCH contributed new reagents/analytical tools, analysed and interpreted data, contributed to discussion and reviewed/edited the manuscript; DGP designed research, analyzed and interpreted data, contributed to discussion and reviewed/edited the manuscript; FKG designed research, obtained funding, supervised the study, analysed and interpreted data, wrote and reviewed/edited the manuscript. All authors have approved the final version of the manuscript.

Duality of interest The authors declare that there is no duality of interest associated with this manuscript.

\section{References}

1. Leslie RD, Delli Castelli M (2004) Age-dependent influences on the origins of autoimmune diabetes: evidence and implications. Diabetes 53:3033-3040

2. Pipeleers D, Ling Z (1992) Pancreatic beta cells in insulindependent diabetes. Diabetes Metab Rev 8:209-227

3. Gorus FK (1997) Diabetes registries and early biological markers of insulin-dependent diabetes mellitus. Belgian Diabetes Registry. Diabetes Metab Rev 13:247-274

4. Skyler JS, Ricordi C (2011) Stopping type 1 diabetes: attempts to prevent or cure type 1 diabetes in man. Diabetes 60:1-8

5. Keymeulen B, Walter M, Mathieu C et al (2010) Four-year metabolic outcome of a randomised controlled CD3-antibody trial in recent-onset type 1 diabetic patients depends on their age and baseline residual beta cell mass. Diabetologia 53:614-623

6. Gorus FK, Pipeleers DG (2001) Prospects for predicting and stopping the development of type 1 of diabetes. The Belgian Diabetes Registry. Best Pract Res Clin Endocrinol Metab 15:371389

7. Vandewalle CL, Coeckelberghs MI, de Leeuw IH et al (1997) Epidemiology, clinical aspects, and biology of IDDM patients under age 40 years. Comparison of data from Antwerp with complete ascertainment with data from Belgium with $40 \%$ ascertainment. The Belgian Diabetes Registry. Diabetes Care 20:1556-1561

8. Kyvik K, Nyström L, Gorus F et al (2004) The epidemiology of type 1 diabetes mellitus is not the same in young adults as in children. Diabetologia 47:377-384

9. Ziegler AG, Hummel M, Schenker M, Bonifacio E (1999) Autoantibody appearance and risk for development of childhood diabetes in offspring of parents with type 1 diabetes: the 2-year analysis of the German BABYDIAB Study. Diabetes 48:460468

10. Kimpimäki T, Kupila A, Hämäläinen A-M et al (2001) The first signs of $\beta$-cell autoimmunity appear in infancy in genetically susceptible children from the general population: the Finnish type 1 prediction and prevention study. J Clin Endocrinol Metab 86:4782-4788

11. Rewers M, Norris JM, Eisenbarth GS et al (1996) Beta-cell autoantibodies in infants and toddlers without IDDM relatives: Diabetes Autoimmunity Study in the Young (DAISY). J Autoimmun 9:405-410

12. Carmichael SK, Johnson SB, Baughcum A et al (2003) Prospective assessment in newborns of diabetes autoimmunity (PANDA): maternal understanding of infant diabetes risk. Genet Med 5:77-83

13. TEDDY Study Group (2008) The Environmental Determinants of Diabetes in the Young (TEDDY) Study. Ann NY Acad Sci 1150:1-13 
14. Knip M, Korhonen S, Kulmala P et al (2010) Prediction of type 1 diabetes in the general population. Diabetes Care 33:1206-1212

15. Simell O, Winter WE, Schatz D (2010) Enhancing the understanding of pre-type 1 diabetes in the general population. Diabetes Care 33:1403-1405

16. De Grijse J, Asanghanwa M, Nouthe B et al (2010) Predictive power of screening for antibodies against insulinoma-associated protein 2 beta (IA-2 $\beta$ ) and zinc transporter- 8 to select first-degree relatives of type 1 diabetic patients with risk of rapid progression to clinical onset of the disease: implications for prevention trials. Diabetologia 53:517-524

17. Truyen I, de Pauw P, Jørgensen P et al (2005) Proinsulin levels and proinsulin-to-C-peptide ratio complement autoantibody measurement for predicting type 1 diabetes. Diabetologia 48:2322-2329

18. Van der Auwera BJ, Schuit FC, Weets I et al (2002) Relative and absolute HLA-DQA1-DQB1 linked risk for developing type I diabetes before 40 years of age in the Belgian population: implications for further prevention studies. The Belgian Diabetes Registry. Hum Immunol 63:40-50

19. Wenzlau JM, Juhl K, Yu L et al (2007) The cation efflux transporter ZnT8 (Slc30A8) is a major autoantigen in human type 1 diabetes. Proc Natl Acad Sci USA 104:17040-17045

20. Achenbach P, Bonifacio E, Koczwara K, Ziegler A (2005) Natural history of type 1 diabetes. Diabetes 53(Suppl 2):S25-S31

21. Jasinski JM, Eisenbarth GS (2005) Insulin as a primary autoantigen for type 1A diabetes. Clin Dev Immunol 12:181-186

22. Bingley PJ (2010) Clinical application of diabetic antibody testing. J Clin Endocrinol Metab 95:25-33

23. Wenzlau JM, Frisch LM, Gardner TJ, Sarkar S, Hutton JC, Davidson HW (2009) Novel antigens in type 1 diabetes: the importance of ZnT8. Curr Diab Rep 9:105-112

24. Myers MA, Rabin DU, Rowley MJ (1995) Pancreatic islet cell cytoplasmic antibody in diabetes is represented by antibodies to islet cell antigen 512 and glutamic acid decarboxylase. Diabetes 44:1290-1295

25. Achenbach P, Lampasona V, Landherr U et al (2009) Autoantibodies to zinc transporter 8 and SLC $30 A 8$ stratify type 1 diabetes risk. Diabetologia 52:1881-1888

26. Savola K, Läärä E, Vähäsalo $P$ et al (2001) Dynamic pattern of disease-associated autoantibodies in siblings of children with type 1 diabetes. Diabetes 50:2625-2632

27. Barker JM, Barriga KJ, Yu L et al (2004) Prediction of autoantibody positivity and progression to type 1 diabetes:
Diabetes Autoimmunity Study in the Young (DAISY). J Clin Endocrinol Metab 89:3896-3902

28. Gorus FK, Vandewalle CL, Dorchy H, van Crombrugge P, Schuit FC, Pipeleers DG (1994) Influence of age on the associations among insulin autoantibodies, islet cell antibodies, and HLA DAQ1*0301-DQB1*0302 in siblings of patients with type 1 (insulin-dependent) diabetes mellitus. Belgian Diabetes Registry. $\mathrm{J}$ Clin Endocrinol Metab 36:1155-1162

29. Yu J, Yu L, Bugawan TL et al (2000) Transient antiislet autoantibodies: infrequent occurrence and lack of association with "genetic" risk factors. J Clin Endocrinol Metab 85:24212428

30. Truyen I, de Grijse J, Weets I et al (2007) Identification of prediabetes in first-degree relatives at intermediate risk of type I diabetes. Clin Exp Immunol 149:243-250

31. von Herrath M, Sanda S, Herold K (2007) Type 1 diabetes as a relapsing-remitting disease? Nat Rev Immunol 7:988-994

32. Savola K, Bonifacio E, Sabbah E et al (1998) IA-2 antibodies: a sensitive marker of IDDM with clinical onset in childhood and adolescence. Diabetologia 41:424-429

33. Decochez K, de Leeuw I, Keymeulen B et al (2002) IA-2 autoantibodies predict impending type 1 diabetes in siblings of patients. Diabetologia 45:1658-1666

34. Pihoker C, Gilliam LK, Hampe CS, Lernmark $\AA$ (2005) Autoantibodies in diabetes. Diabetes 54(Suppl 2):S52-S61

35. Achenbach P, Warncke K, Reiter J et al (2006) Type 1 diabetes risk assessment: improvement by follow-up measurements in young islet autoantibody-positive relatives. Diabetologia 49:29692976

36. Vehik K, Haller MJ, Beam CA et al (2011) Islet autoantibody seroconversion in the DPT-1 study. Diabetes Care 34:358362

37. Vehik K, Beam CA, Mahon JL et al (2011) Development of autoantibodies in the TrialNet natural history study. Diabetes Care 34:1897-1901

38. Dahlquist G, Mustonen LR (1995) Clinical onset characteristics of familial versus nonfamilial cases in a large population-based cohort of childhood-onset diabetes patients. Diabetes Care $18: 852-854$

39. Veijola $\mathrm{R}$, Reijonen $\mathrm{H}$, Vähäsalo $\mathrm{P}$ et al (1996) $H L A-D Q B 1$ defined genetic susceptibility, beta cell autoimmunity and metabolic characteristics in familial and nonfamilial insulin-dependent diabetes mellitus. Childhood Diabetes in Finland (DiMe) Study Group. J Clin Invest 96:2489-2495 